\title{
Ultracold neutral plasma expansion in two dimensions
}

\author{
E. A. Cummings \\ J. E. Daily \\ Dallin S. Durfee \\ Scott D. Bergeson \\ scott.bergeson@byu.edu
}

Follow this and additional works at: https://scholarsarchive.byu.edu/facpub

Part of the Astrophysics and Astronomy Commons, and the Physics Commons

\section{Original Publication Citation}

Cummings, E. A., J. E. Daily, D. S. Durfee, and S. D. Bergeson."Ultracold neutral plasma expansion in two dimensions." Physics of Plasmas 12 (25).

\section{BYU ScholarsArchive Citation}

Cummings, E. A.; Daily, J. E.; Durfee, Dallin S.; and Bergeson, Scott D., "Ultracold neutral plasma expansion in two dimensions" (2005). Faculty Publications. 332.

https://scholarsarchive.byu.edu/facpub/332 


\title{
Ultracold neutral plasma expansion in two dimensions
}

\author{
E. A. Cummings, ${ }^{\text {a) }}$ J. E. Daily, D. S. Durfee, and S. D. Bergeson ${ }^{\text {b) }}$ \\ Department of Physics and Astronomy, Brigham Young University, Provo, Utah 84602
}

(Received 14 September 2005; accepted 3 November 2005; published online 12 December 2005)

An isothermal model of ultracold neutral plasma expansion is extended to systems without spherical symmetry. It is used to interpret new fluorescence measurements on ultracold neutral calcium plasmas. For a self-similar expansion, the fluid equations are solved both analytically and numerically. The density and velocity solutions are used to predict fluorescence signals induced by a laser beam weakly focused into the plasma. Despite the simplicity of the model, predicted fluorescence signals reproduce major features of the experimental data. (C) 2005 American Institute of Physics. [DOI: 10.1063/1.2140683]

\section{INTRODUCTION}

Ultracold plasmas are produced from photoionized lasercooled gases. ${ }^{1-3}$ In these laboratory plasmas, it is possible to study the kinetics and thermodynamics of multicomponent, strongly interacting Coulomb systems. These systems are characterized by the ratio of the nearest-neighbor Coulomb energy to the average kinetic energy, denoted as $\Gamma$ $=\left(q^{2} / 4 \pi \epsilon_{0} d\right) /\left(k_{B} T\right)$, where $q$ is the quantum of electrical charge, $\epsilon_{0}$ is the permittivity of free space, $d$ is the interparticle spacing, $k_{B}$ is Boltzmann's constant, and $T$ is the equilibrium temperature.

A new class of ultracold plasma experiments has recently become available in which it is possible to spectroscopically study the plasma ions. ${ }^{4-6}$ These plasmas are made using alkaline-earth atoms, because the resonance transition wavelengths of the ions are readily generated using standard laser methods. The spatially resolved time evolution of the plasma ion temperature and density can be measured using absorption and fluorescence techniques.

These ultracold neutral plasmas are not trapped, although efforts are underway in a few laboratories to trap them. The untrapped plasmas freely expand, and as they expand the density and temperature change radically. Processes of recombination, collisional and thermal ionization, radiative cascade, and adiabatic and evaporative cooling all play important roles in how the system evolves and equilibrates.

A variety of models have been used to investigate the properties of these plasmas. ${ }^{7-13}$ One particularly simple isothermal fluid model $^{7}$ has been surprisingly successful in predicting the general features of these plasmas. ${ }^{6,8,12}$ In this paper we extend this model from the spherically symmetric Gaussian plasma distributions to Gaussian distributions with elliptical symmetry.

The elliptical symmetry has important experimental advantages. In such systems the plasma expands primarily in two dimensions. The practical advantage is that the density falls more slowly than in the three-dimensional case, making it possible to study the plasma for longer times. The Doppler

\footnotetext{
a) Present address: Lockheed Martin Space Systems Company, Sunnyvale, CA 94089.

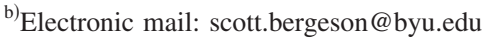

shift due to the directed expansion of the plasma is also suppressed. It should therefore be possible to study plasma oscillations and heating effects for greater time periods before these oscillations are masked by the directed expansion of the plasma. Finally, if the plasmas are generated from a density-limited neutral atom trap, the elongated symmetry allows a greater number of atoms to be trapped initially, corresponding to a greater column density of plasma ions. This directly increases the visibility of fluorescence and absorption signals.

\section{ISOTHERMAL FLUID MODEL}

An isothermal fluid model has been presented in the literature. ${ }^{7,8}$ It successfully reproduces most of the major features of recent experimental work. This model was motivated by trends observed in more sophisticated treatments. The basic ideas of the model will be reviewed here, and an extension to the case of a Gaussian distribution with elliptical symmetry will be presented.

The initial ion-density distribution is proportional to the Gaussian distribution of the neutral atom cloud from which the plasma is created. For a spherically symmetric cloud, the initial density distribution can be written as $n(r)=n_{0} \exp \left(-\beta r^{2}\right)$, where $n_{0}$ is the peak density and $\beta^{-1 / 2}$ is the time-dependent half-width of the Gaussian distribution. Because the electrons thermalize much faster than the ions, in this model we take the initial electron-density distribution to be the thermal equilibrium distribution, given by the Boltzmann factor:

$$
n_{e}(r)=n_{0 e} \exp \left[\frac{-q V(r)}{k_{B} T_{e}}\right],
$$

where $n_{0 e}$ is the peak initial electron density, $V(r)$ is the electrical potential, and $T_{e}$ is the electron temperature. The lowest-temperature plasmas are nearly charge neutral, and the electron-density distribution is approximately equal to the ion density. In this limit, it is shown in Refs. 7 and 8 that for a spherically symmetric plasma, the electrical potential energy can be written as 


$$
q V(r)=k_{B} T_{e} \ln \left[n(r) / n_{0 e}\right]=-k_{B} T_{e} \beta r^{2}
$$

plus an arbitrary additive constant. The force is the negative gradient of this potential energy. It is manifestly radial, spherically symmetric, and linearly proportional to the radial coordinate $r$, measured from the center of the plasma. The velocity, which is the time integral of the acceleration, is also linearly proportional to $r$. The consequence is that if the distribution is Gaussian initially, it will remain Gaussian at all times in the expansion.

For the case of nonspherical symmetry, the approach is more or less the same. We will take the initial ion-density distribution to be Gaussian, symmetric in the $x-y$ plane, and initially elongated in the $z$ direction:

$$
n_{i}(x, y, z, t)=\frac{N_{i} \beta_{1} \beta_{2}^{1 / 2}}{\pi^{3 / 2}} \exp \left[-\left(x^{2}+y^{2}\right) \beta_{1}-z^{2} \beta_{2}\right] .
$$

In this equation, $n_{i}$ is the ion density and $N_{i}$ is the number of ions in the distribution. As before, the functions $\beta_{1}$ and $\beta_{2}$ are time dependent, and $\beta_{k}^{-1 / 2}$ is the half-width of the distribution with $k=1$ or 2 . The initial conditions are $\beta_{1}(0)=\sigma_{0}^{-2}$ and $\beta_{2}(0)=\alpha^{-2} \sigma_{0}^{-2}$. The parameter $\alpha$ defines the ellipticity of the system, and for the work presented here $\alpha=2.5$.

Neglecting collisions between the ions and electrons, the plasma fluid equations are written as

$$
\begin{aligned}
& \frac{\partial n}{\partial t}+\nabla \cdot(n \mathbf{v})=0, \\
& \frac{\partial \mathbf{v}}{\partial t}+(\mathbf{v} \cdot \nabla) \mathbf{v}=\mathbf{a} .
\end{aligned}
$$

Following the derivation used in the case of spherical symmetry, the velocity is taken to be $\mathbf{v}=\gamma_{1}(t)(x \hat{x}+y \hat{y})+\gamma_{2}(t) z \hat{z}$. Inserting this into Eq. (4) and using the density profile of Eq. (3) give

$$
\begin{aligned}
& 2 \beta_{2}\left(x^{2} \beta_{1}+y^{2} \beta_{1}-1\right)\left(\dot{\beta}_{1}+2 \beta_{1} \gamma_{1}\right)+4 \beta_{1}\left(z^{2} \beta_{2}-1\right)\left(\dot{\beta}_{2}\right. \\
& \left.\quad+2 \beta_{2} \gamma_{2}\right)=0 .
\end{aligned}
$$

Because $x, y$, and $z$ are independent variables, the only nontrivial solution of this equation is

$$
\gamma_{k}=-\dot{\beta}_{k} / 2 \beta_{k},
$$

where $k=1$ or 2 . The equation is identical for expansion in the $x-y$ plane or in the $z$ direction.

The ion acceleration in Eq. (5) is calculated by dividing the force (the negative gradient of the electrical potential energy) by the ion mass $m_{i}$,

$$
\mathbf{a}=\frac{k_{B} T_{e}}{m_{i}}\left(2 x \beta_{1} \hat{x}+2 y \beta_{1} \hat{y}+2 z \beta_{2} \hat{z}\right) .
$$

Then Eq. (5) is written as

$$
\dot{\gamma}_{k}+\gamma_{k}^{2}=2 k_{B} T_{e} \beta_{k} / m_{i},
$$

where $k=1$ or 2 . Once again this expansion equation is identical for the two expansion directions. The conservation of energy relates the electron temperature to the $\gamma$ 's and $\beta$ 's,

$$
T_{e}(t)=T_{e}(0)-\frac{m_{i}}{3 k_{B}}\left(\frac{\gamma_{2}^{2}}{2 \beta_{2}}+\frac{\gamma_{1}^{2}}{\beta_{1}}\right),
$$

where $T_{e}(0)$ is the initial electron temperature at time $t=0$. In this expression, the kinetic energy is obtained by integrating $m v^{2} / 2$ over the density distribution, and we have neglected the energy due to electron-ion recombination which is only important at the lowest electron energies. ${ }^{2,7,8}$

Equations (7), (9), and (10) are a complete set of coupled equations. Equations (7) and (9) are first order in time and can be solved numerically. Analytic solutions to these equations can be derived if we make the additional assumption that the electron temperature is isothermal in a given dimension but anisotropic in space. Although this might seem overly simplistic, it does give the proper behavior in the limiting case of a plasma infinitely long in the $z$ dimension. This decoupling has the consequence that energy is to be conserved separately in the $x-y$ plane and in the $z$ dimension.

In this limit, Eq. (10) can be written as

$$
T_{k e}(t)=T_{e}(0)-\frac{m}{2 k_{B}} \frac{\gamma_{k}^{2}}{\beta_{k}} .
$$

The plasma equations for these two spaces, $k=1$ or $k=2$, are now exactly identical and completely separable. Equations (7), (9), and (11) are the same as Eq. (2) of Ref. 7, with slightly different boundary conditions for $\beta_{1}$ and $\beta_{2}$. Using Eqs. (7) and (10), we now have both $T_{k e}$ and $\gamma_{k}$ in terms of $\beta_{k}$. Inserting this into Eq. (9) gives

$$
\frac{\ddot{\beta}_{k}}{\beta_{k}^{2}}-2 \frac{\dot{\beta}_{k}^{2}}{\beta_{k}^{3}}+2 v_{e}^{2}=0,
$$

where we have made the substitution $v_{e}^{2}=2 k_{B} T_{e}(0) / m_{i}$. With this definition, $v_{e}$ represents the expansion velocity of ions in the plasma. If there was no expansion, it would be the ion velocity in thermal equilibrium. The solution to this equation is $\beta_{1}^{-1}=\sigma_{0}^{2}+c t+v_{e}^{2} t^{2}$, where $c$ is an integration constant. The constant $c$ must be zero to meet the condition that the ion velocity is initially zero at $t=0 .{ }^{14}$ For this case, in which we assume temperature decoupling, the $\beta$ 's and $\gamma$ 's can be written as

$$
\begin{aligned}
& \beta_{1}(t)=\left(\sigma_{0}^{2}+v_{e}^{2} t^{2}\right)^{-1}, \\
& \beta_{2}(t)=\left(\alpha^{2} \sigma_{0}^{2}+v_{e}^{2} t^{2}\right)^{-1}, \\
& \gamma_{1}(t)=v_{e}^{2} t \beta_{1}(t), \\
& \gamma_{2}(t)=v_{e}^{2} t \beta_{2}(t) .
\end{aligned}
$$

A comparison between these analytical solutions and the numerical solutions of the plasma equations is shown in Fig. 1. For expansion in the $x-y$ plane, the decoupled temperature approximation makes essentially no difference. However, in the $z$ direction the plasma accelerates less and expands more slowly. This slower acceleration and expansion in $z$ mean that the fluorescence signal will fall more slowly at late times.

We note that for two-dimensional planar Gaussian charge distributions, a closed-form expression for the electric 


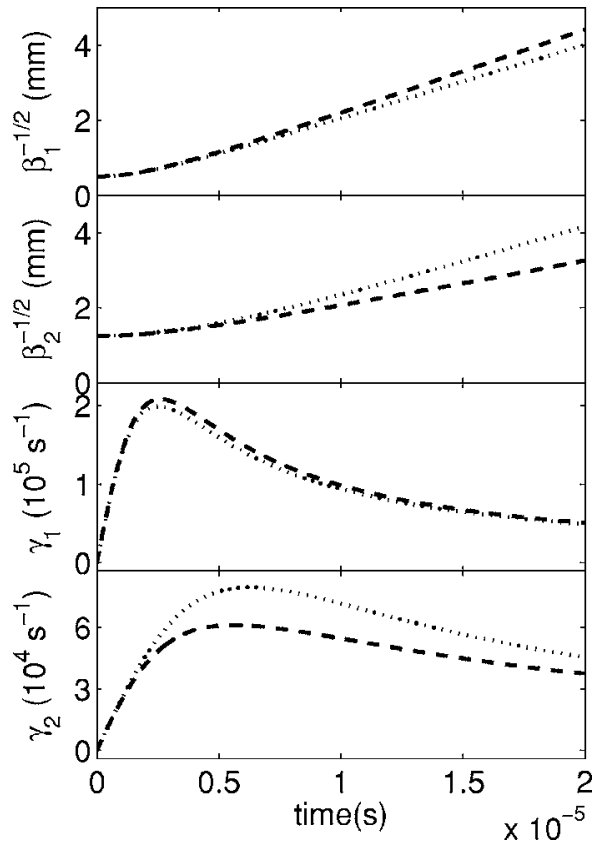

FIG. 1. A comparison between numerical (dashed lines) and analytical (dotted lines) solutions of the plasma equations for the $\beta$ 's and $\gamma$ 's. The conditions for these plots are $E_{e}=100 \mathrm{~cm}^{-1}, \sigma_{0}=0.5 \mathrm{~mm}$, and $\alpha=2.5$, typical parameters in the experiment. The numerical solutions show that the plasma expands more quickly in the $x-y$ plane and more slowly in the $z$ dimension than the analytical solutions predict. However, these differences have a relatively small influence on the predicted fluorescence signals.

field has been derived. ${ }^{15}$ If such a compact analytical solution could be written in the three-dimensional case, it would remove the decoupling constraint that we imposed in order to find analytic solutions to the plasma equations. However, such a solution is not readily apparent.

\section{FLUORESCENCE SIGNAL MODEL}

The geometry of our measurements is shown in Fig. 2. In the experiment, the probe laser-beam is spatially filtered and focused into the plasma with a confocal beam parameter that is long compared to all plasma dimensions. The position offset of this probe laser relative to the plasma is denoted by the parameter $a$. After the plasma is created, the number of atoms in the column defined by the probe-laser beam changes dramatically. When the laser beam passes through the center of the plasma $(a=0)$, the number of ions in the beam monotonically decreases. However, if the laser is outside of the initial plasma distribution, as shown in Fig. 2, the number first increases as the plasma moves into the laser beam and then decreases as the plasma disperses.

The fluorescence signal depends on both the number of ions in the column defined by the probe-laser beam (Gaussian beam profile with $1 / e^{2}$ radius $w$ ) and on the velocity distribution of the ions in the plasma. Because the laser has a narrow bandwidth, atoms moving at velocities greater than $\sim 4.5 \mathrm{~m} / \mathrm{s}$ are Doppler shifted out of resonance. In this section we will use the results of the previous section to derive an expression for how the plasma ion fluorescence signal should change with time for different values of the offset parameter $a$.

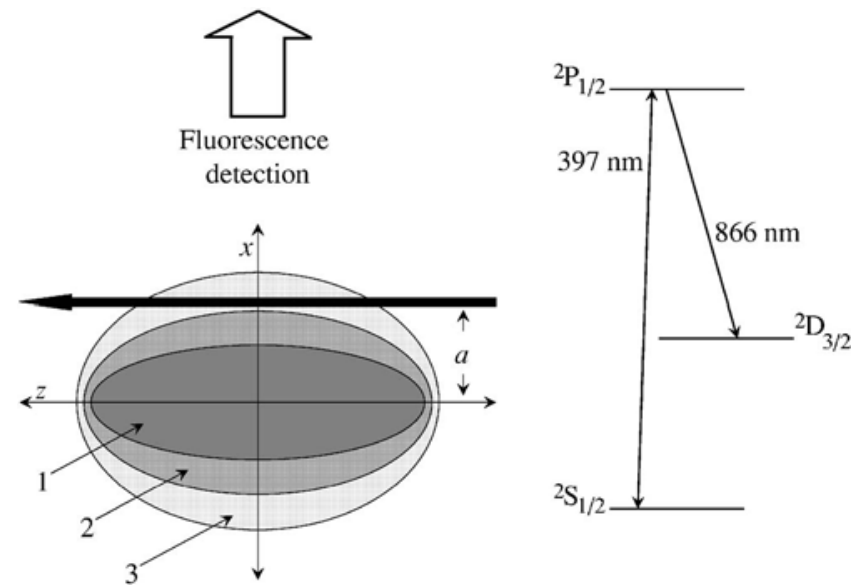

FIG. 2. A schematic diagram of the fluorescence measurements. Left-the plasma is initially at a high density and elongated in the $z$ dimension (labeled as region 1). The probe laser is represented by the solid dark arrow parallel to the $z$ axis, displaced by a variable distance $a$. At later times the plasma expands slowly in $z$ but quickly in the $x-y$ plane. Regions 2 and 3 represent the plasma size at later times. As the plasma expands, the number of atoms in the column defined by the probe laser changes. Right - a partial level diagram of $\mathrm{Ca}^{+}$. The probe laser is locked to the $397 \mathrm{~nm}$ resonance transition, and we measure $397 \mathrm{~nm}$ light scattered by plasma ions.

The fluorescence signal $\mathcal{S}(t)$ is proportional to the absorption of the probe-laser beam. Using Beer's law and the standard approximation of small optical depth, $\mathcal{S}(t)$ can be written as

$$
\mathcal{S}(t) \propto \int_{\mathrm{vol}} n_{i}(x, y, z, t) f_{1}(x-a, y) \bar{\sigma}\left(\nu-\nu_{0}\right),
$$

where $f_{1}$ is the spatial profile of the probe-laser beam and $\bar{\sigma}$ is the absorption cross section as a function of $\nu-\nu_{0}$, the difference between the laser frequency and the atomic resonance frequency. When the optical depth is not small, the fluorescence signal is proportional to $1-\exp [\mathcal{S}(t)]$. For the experiments presented here, the maximum initial optical depth for the probe-laser beam is around 0.8, but it quickly falls to 0.3 at $50 \mathrm{~ns}$ because of plasma heating. However, because the fluorescence measurements are made with the probe-laser beam intensity several times greater than the saturation intensity of the ion transition, the effective optical depth for the probe-laser beam is reduced. The optical depth for the scattered photons is also small because of the elongated geometry of the initial distribution.

We can simplify Eq. (17) by setting the laser frequency equal to $\nu_{0}$ and recognizing $\nu-\nu_{0}=v_{z} / \lambda$ as the Doppler shift due to the atom's velocity component $v_{z}$ along the direction of the laser-beam propagation, with $\lambda$ being the optical wavelength of the transition. We write $v_{z}=z \gamma_{2}$ and define a length $\ell \equiv \lambda \Gamma_{n} / 2 \gamma_{2}$, where $\Gamma=1 / 2 \pi \tau$ is the natural width of the transition and $\tau$ is the lifetime of the transition's upper state, and use it to write the absorption profiles,

$$
\bar{\sigma} / \bar{\sigma}_{0}= \begin{cases}{\left[(z / \ell)^{2}+1+s\right]^{-1},} & \text { Lorentzian } \\ \exp \left[-2\left(v_{\mathrm{th}} / 2 \Gamma_{n} \lambda\right)^{2}(z / \ell)^{2}\right], & \text { Gaussian, }\end{cases}
$$

where $\bar{\sigma}_{0}$ is the absorption cross section on resonance, $v_{\text {th }}$ is the rms velocity of a thermal distribution, and $s$ is the satu- 


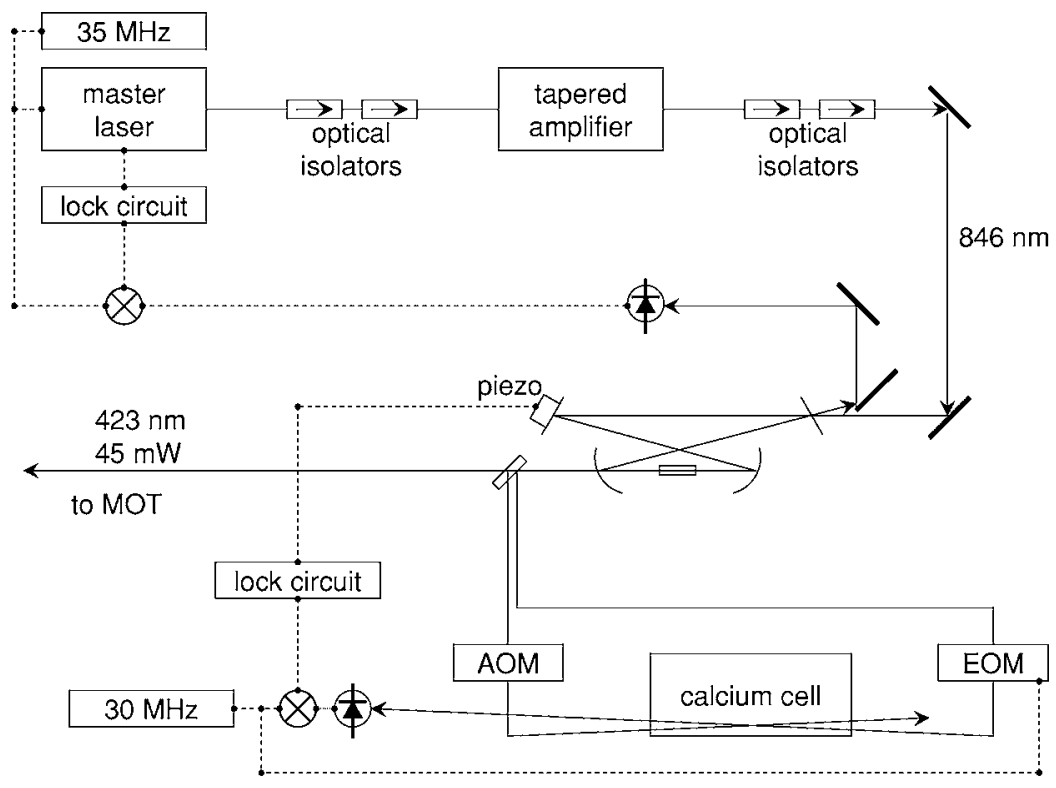

FIG. 3. A schematic drawing of the MOT laser system and frequency stabilization electronics used in these experiments. $\mathrm{AOM}=$ acousto-optical modulator. EOM =electro-optical modulator.

ration parameter, defined as the probe-laser intensity divided by the saturation intensity. ${ }^{16}$ The $z$ dependence in these expressions comes from the result of the thermal model that links the $z$ velocity component with the $z$ position. The length $\ell$ is $z$ position at which the Doppler shift due to the directed velocity of the plasma ions is equal to the natural linewidth. The true absorption line shape is better represented by a Voigt profile. We note that the Voigt profile can be approximated by a linear combination of the Lorentzian and Gaussian line profiles. ${ }^{17}$ For simplicity, the remainder of this derivation will only consider the Lorentzian line shape.

We take the plasma density profile from Eq. (3) and write the spatial profile of the probe-laser beam as

$$
f_{1}=\exp \left[-2(x-a)^{2} / w^{2}-2 y^{2} / w^{2}\right]
$$

which corresponds to the geometry represented in Fig. 2. Performing the integration in Eq. (17) gives

$$
\mathcal{S}(t) \propto \frac{\xi \eta}{a^{2}(1+s)} \exp \left(\eta^{2}-\xi\right) \operatorname{erfc}(\eta),
$$

where $\xi=2 a^{2} \beta_{1}^{2} /\left(\beta_{1}^{2} w^{2}+2\right)$ and $\eta=\ell \sqrt{\beta_{2}(1+s)}$. In the limit of low probe-laser intensity $(s \rightarrow 0)$ and for the case in which the temperatures in the $x-y$ plane and the $z$ dimension decouple, this expression for the fluorescence signal is proportional to Eq. (5) of Ref. 6.

\section{ULTRACOLD CALCIUM PLASMAS}

We create ultracold neutral plasmas by photoionizing laser-cooled calcium atoms in a magneto-optical trap (MOT). The calcium MOT is formed in the usual way by three pairs of counterpropagating laser beams that intersect at right angles in the center of a magnetic quadrupole field. ${ }^{18}$ The $423 \mathrm{~nm}$ laser light required for the calcium MOT is generated by frequency doubling an infrared laser in periodically poled potassium titanyl phosphate (PPKTP). ${ }^{19}$ A diode laser master-oscillator-power-amplifier (MOPA) system delivers $300 \mathrm{~mW}$ single frequency at $846 \mathrm{~nm}$, as shown in Fig. 3. This laser is phase locked to a buildup cavity using the
Pound-Drever-Hall technique, ${ }^{20}$ giving a power enhancement of 30. A 20-mm-long PPKTP crystal in the small waist of the buildup cavity is used to generate typically $45 \mathrm{~mW}$ output power at $423 \mathrm{~nm}^{21,22}$

The laser is further stabilized by locking the $423 \mathrm{~nm}$ light to the calcium resonance transition using saturated absorption spectroscopy in a calcium vapor cell. ${ }^{23}$ Our vapor cell differs from Ref. 23 in that it has a stainless-steel body with conflat metal seals and conflat-mounted windows and a valve. An acousto-optic modulator (AOM) in one arm of the saturated absorption laser beams shifts the laser frequency so that the laser beam sent to the MOT is $35 \mathrm{MHz}$ (one natural linewidth) below the atomic resonance. We also use the AOM to chop this beam and use a lock-in amplifier to eliminate the Doppler background in the saturated absorption signal. Because the $846 \mathrm{~nm}$ laser is already locked to the frequency-doubling cavity, the feedback from this second lock circuit servos the frequency-doubling cavity length.

The trap is loaded from a thermal beam of calcium atoms that passes through the center of the MOT. The thermal beam is formed by heating the calcium in a stainless-steel oven to $650{ }^{\circ} \mathrm{C}$. The beam is weakly collimated by a 1-mm-diameter, 10-mm-long aperture in the oven wall. The distance between the oven and the MOT is approximately $10 \mathrm{~cm}$. As the beam passes through the MOT, the slowest atoms in the velocity distribution are cooled and trapped. An additional red-detuned $(140 \mathrm{MHz}$, or four times the natural linewidth) laser beam counterpropagates the calcium atomic beam, significantly enhancing the MOT's capture efficiency. To prevent optical pumping of the neutral calcium MOT atoms into metastable dark states, we also employ a diode laser at $672 \mathrm{~nm}$ which is locked to a transition in neutral calcium. The density profile of the MOT has an asymmetric Gaussian profile and at time $t=0$ is well represented by Eq. (3) with the peak density equal to $4 \times 10^{9} \mathrm{~cm}^{-3}, \sigma_{0}=0.5 \mathrm{~mm}$, and $\alpha=2.5$.

We photoionize the atoms in the MOT using a two-color, two-photon ionization process. A portion of the $846 \mathrm{~nm}$ di- 

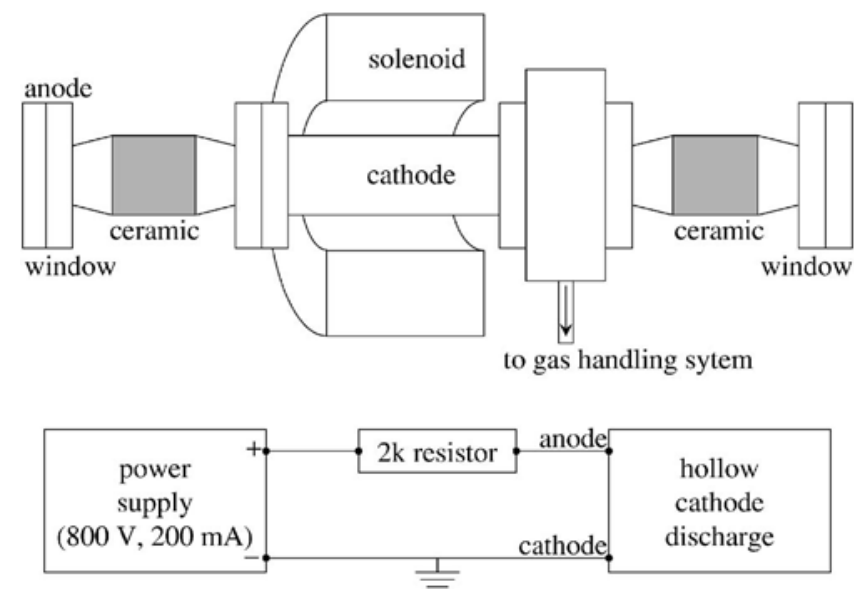

FIG. 4. A schematic diagram of the DAVLL cell. Upper-a drawing of the hollow-cathode discharge cell, approximately to scale. The cathode is $10 \mathrm{~cm}$ long, with a 1-cm-diameter bore. It is machined out of solid calcium and press fit into the conflat nipple that serves as the cathode. The operating pressure is between 100 and 200 mTorr of krypton. Lower-electrical connections to the discharge cell. For increased current stability, a $2 \mathrm{k} \Omega$ ballast resistor is connected in series with the discharge.

ode laser radiation from the MOT laser is pulse amplified in a pair of yttrium aluminum garnet (YAG)-pumped dye cells and frequency doubled. This produces a 3 ns duration laser pulse at $423 \mathrm{~nm}$ with a pulse energy around $1 \mu \mathrm{J}$. This laser pulse passes through the MOT, and its peak intensity is a few thousand times greater than the saturation intensity. A second YAG-pumped dye laser at $390 \mathrm{~nm}$ counterpropagates the $423 \mathrm{~nm}$ pulse and excites the MOT atoms to low-energy states in the region of the ionization potential. We photoionize $85 \%-90 \%$ of the ground-state atoms in the MOT. The minimum initial electron temperature is limited by the bandwidth of the $390 \mathrm{~nm}$ laser to about $1 \mathrm{~K}$.

Ions in the plasma scatter light from a probe-laser beam tuned to the $\mathrm{Ca} \mathrm{II}{ }^{2} S_{1 / 2}-{ }^{2} P_{1 / 2}$ transition at $397 \mathrm{~nm}$. The probe laser is generated by a grating-stabilized violet diode laser. This laser, as well as the $672 \mathrm{~nm}$ laser used in the neutral atom trap, is locked using the dichroic-atomic-vapor laser lock (DAVLL) technique. ${ }^{24} \mathrm{~A}$ vapor of ions and excited-state neutral atoms for these two locks is generated in a largebore, low-pressure hollow-cathode discharge of our own design (see Fig. 4).

The probe laser is spatially filtered. The typical probelaser intensity is a few hundred microwatts focused to a Gaussian waist of $130 \mu \mathrm{m}$ in the MOT. The peak probe-laser intensity is a few times the saturation intensity of the $397 \mathrm{~nm}$ transition. We average repeated measurements of the scattered $397 \mathrm{~nm}$ radiation with the probe laser in a given position, denoted by the parameter $a$ in Fig. 2. This produces a time-resolved signal proportional to the number of atoms resonant with the probe beam in a particular column of the plasma. By translating a mirror just outside the MOT chamber, we scan the probe laser across the ion cloud. In this manner we obtain temporal and spatial informations about the plasma expansion.

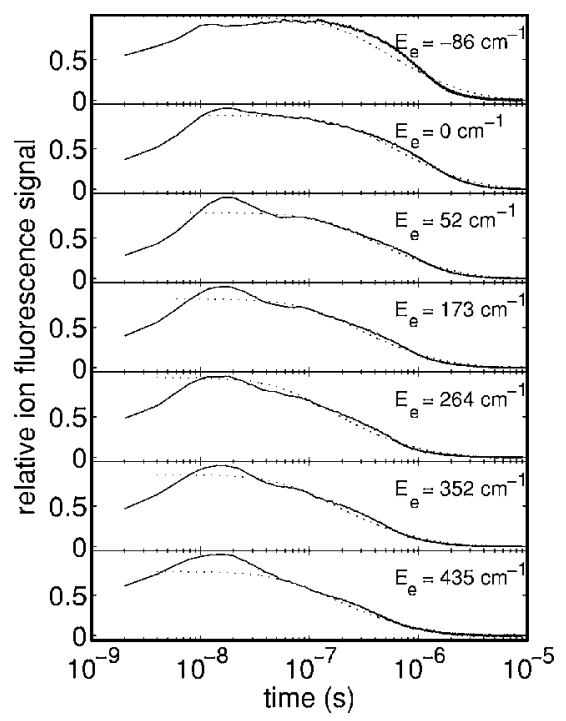

FIG. 5. Relative ion fluorescence signal. The probe laser is tuned to resonance and propagates through the center of the plasma $(a=0)$. The electron energy $E_{e}$ is measured relative to the ionization limit. The solid line is the fluorescence signal; the dotted line is a fit using the Lorentzian line shape in Eq. (20). The model is normalized to the fluorescence signal at $t=10^{-7} \mathrm{~s}$, and the single fitting parameter is the expansion velocity $v_{e}$. In the top panel, the plasma is spontaneously generated after exciting the atoms to a Rydberg state with $n^{*} \sim 35.7$

\section{COMPARING THE MODEL TO THE DATA}

One comparison of the isothermal model with experimental data was presented in Ref. 6. In that work, the initial electron energy of the plasma, and therefore the expansion velocity $v_{e}$, was fixed, and the parameter $a$ was varied from 0 to $4 \sigma_{0}$.

In the following, we present a complementary comparison. The solid line in Fig. 5 shows ion fluorescence signal with the probe laser tuned to the ion resonance frequency, and with the probe-laser beam propagating along the $z$ axis ( $a=0$ in Fig. 2) for a range of initial electron energies.

As discussed in Ref. 6, the early rise in the fluorescence signal shows the increasing number of plasma ions in the ${ }^{2} P_{1 / 2}$ level. This feature can be explained in terms of the classic Rabi two-level atom with damping. The probe-laser intensity is in the range of 3-5 times the resonance Rabi frequency. Our numerical integration of the optical Bloch equations mimics the approximately $20 \mathrm{~ns}$ rise time observed in the experimental data and shows that the Gaussian spatial profile of the probe-laser beam washes out subsequent oscillations in the excited-state fraction.

Following the initial rise, the fluorescence decays. At approximately $t=50 \mathrm{~ns}$ the decay slows. This behavior is due to correlation-induced heating in the plasma, as discussed in the literature. ${ }^{4,6}$ Correlations heat the plasma ions for times less than the inverse ion plasma frequency. This is the time required for the ions to reach a local equilibrium. While the ions are heated, their velocity distribution increases, which Doppler shifts a part of the distribution out of resonance with the probe-laser beam. Once a local equilibrium is reached, correlation heating turns off.

These two processes are not included in the isothermal 
model. We therefore begin the comparison of the model with the data at time $t=10^{-7} \mathrm{~s}$. The model is normalized to the data and fitted using $v_{e}$ as the only fit parameter in an unweighted least-squares-fitting routine. For the $a=0$ data, the analytical and numerical solutions give essentially indistinguishable fluorescence line shape predictions. The model uses the Lorentzian line shape, as derived in Eq. (20). The justification for doing so comes from the analysis presented in Ref. 6. In Fig. 2 of that reference, the $t=0$ velocity is $6 \mathrm{~m} / \mathrm{s}$. This is the $z$ component of the rms velocity of a thermal distribution, and it is due almost entirely to correlation-induced heating. This velocity width gives a Doppler width comparable to the natural linewidth, and the Voigt profile is close to a pure Lorentzian. Furthermore, the saturation parameter is around $s=3$, which broadens the Lorentzian width by $\sqrt{3}$, making the Doppler width even less important. Finally, as the plasma evolves, the ion temperature probably falls due to adiabatic expansion. The time scale for this is $\sim \sigma_{0} / v_{e}=2 \mu \mathrm{s}$. This is shorter than the time scale for heating the ions by collisions with the electrons, $\sim m_{i} / m_{e} \omega_{p}=50 \mu \mathrm{s}$. The figure shows that the Lorentzian model describes the data well over a few orders of magnitude in time. The velocities extracted from these data are shown in Fig. 7.

The differences between the model and the signal are not negligible. For all initial electron energies, the model is slightly too low at $t=300 \mathrm{~ns}$ and too high at $t>1 \mu \mathrm{s}$. The data are not corrected for optical pumping into the $D$ states (see Fig. 2). This could explain part of the missing signal at $t>1 \mu \mathrm{s}$ but not at $t=300 \mathrm{~ns}$. By measuring the fluorescence at different probe-laser intensities, we can determine the importance of optical pumping. To within the noise level on the data, the ratio of the fluorescence signal measured with a high-intensity probe-laser beam to that measured with a lowintensity probe-laser beam is a single exponential decay of the form $\exp [-t /(5 \mu \mathrm{s})]$.

Differences between the model and the fluorescence signal at $t<1 \mu$ s could indicate internal heating processes that manifest themselves in the ion velocity on the few hundred nanosecond time scale. They could also indicate ions that appear in the plasma from Rydberg states at late times or collective plasma density variations. These differences can be studied in future work.

We also compare the fluorescence signal and the model over a range of initial electron energies with the probe-laser beam shifted to $a=1.1 \mathrm{~mm}=2.2 \sigma_{0}$. In this arrangement, the ion fluorescence signal is initially small and grows as ions move into the probe-laser beam. Typical data are shown in Fig. 6. The analytical and numerical solutions are qualitatively the same. However, because the numerical solutions give a slower acceleration and expansion in $z$, the signals fall more slowly (or alternatively, the relative fluorescence signals at early times are smaller). This slower fluorescence decay shifts the apparent peak in the fluorescence model to longer times by about $7 \%$.

When the electron energy $E_{e} \geqslant 173 \mathrm{~cm}^{-1}$, the numerical solutions give a fluorescence line shape in somewhat better agreement with the data. In these data, optical pumping is less important because the ions spend less time in the laser

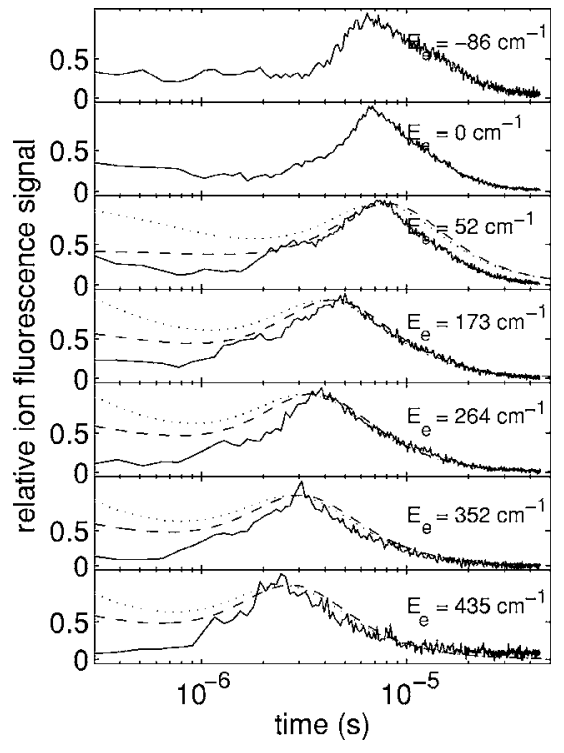

FIG. 6. Relative ion fluorescence signal. The probe laser is tuned to resonance and propagates at a distance $a=1.1 \mathrm{~mm}=2.2 \sigma_{0}$ relative to the center of the initial distribution. The solid line is the data. The dotted line is the fluorescence model using the analytic solutions of the plasma equations. The dashed line is the model using the numeric solutions. The velocity used for the model data is calculated from the initial electron energy. The amplitude is the only adjustable parameter used in these plots.

beam. Our Monte Carlo simulations of the experiment suggest that most ions contributing to these fluorescence signals spend less than a few hundred nanoseconds in the probelaser beam, which is less than the optical pumping time. As we did with the $a=0$ data, the analytic model is fitted to the $t>6 \mu$ s signal using a least-squares procedure with $v_{e}$ as the fitting parameter. The velocity extracted from this fit is shown in Fig. 7. It is also possible to fit the data using $v_{e}$ so that the peak in the model coincides with the peak of the fluorescence signal. These velocities are also plotted in the figure.

For these data, the signal in the first microsecond is small compared to the model. Moreover, there are variations in the fluorescence signal that do not appear in the model. This suggests that at the edges of the plasma expansion, the

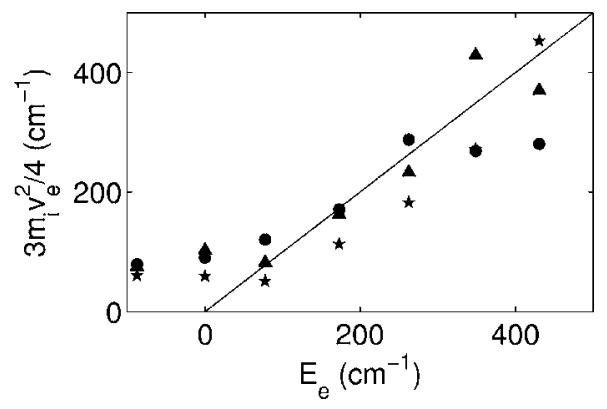

FIG. 7. Expansion energy based on velocity extracted from Figs. 5 and 6. The triangles $(\boldsymbol{\Delta})$ represent the velocity extracted from Fig. 5 and the circles (O) from Fig. 6. The stars $(\star)$ represent the velocity extracted from centering the peak of the model on the peak of the data in Fig. 6. The line shows the relationship $E_{e}=3 m_{i} v_{e}^{2} / 4$. The apparent extra factor of 2 in the denominator comes from the fact that we defined the expansion velocity to be $v_{e}$ $=\sqrt{2 k_{B} T_{e} / m_{i}}$, which is a factor of $\sqrt{2}$ larger than the traditional definition. 
density distribution is non-Gaussian, even at early times before any ion motion is possible. It also appears, as pointed out in Ref. 6, that the Gaussian density profile is recovered at late times for all initial electron energies.

A comment about the extracted velocities is in order. The plasma expansion energy is expected to be equal to the electron energy. As shown in Fig. 7, the expansion energy equals the initial electron energy for data with $E_{e}>50 \mathrm{~cm}^{-1}$. Below this initial electron energy, effects such as recombination and electron-Rydberg scattering dominate the electron energy distribution. ${ }^{2,7,8}$ These effects are manifested in this data for $E_{e}=0$ and $-86 \mathrm{~cm}^{-1}$. The influence of recombination on the fluorescence signal levels is expected to be minimal because Rydberg atoms recombine into states with relatively high principle quantum numbers and with high angular momentum, and the spectral shift and width in the ion core transition are negligible.

\section{CONCLUSION}

We present an extension of the isothermal plasma expansion model of Refs. 7 and 8 for quasi-two-dimensional geometry. We include velocity effects and predict a fluorescence or absorption signal versus time for given initial conditions. The model matches the correct order of magnitude and general features of the fluorescence signal.

It is surprising that the analytical model reproduces the experimental data as well as it does. The isothermal assumption with complete decoupling between the electron energy in the $z$ and the $x / y$ axes seems to be inappropriate for the experimental conditions. The electron collision time is smaller than the electron oscillation time in the plasma, meaning that the electrons should collide several times as they traverse the plasma.

Some of the effects shown in Fig. 1 are masked by the fact that our measurements project the three-dimensional distribution into two dimensions. While the numerical solutions show the plasma expanding more slowly in $z$, it expands somewhat faster in $x$ and $y$, so that the column density is more or less the same as predicted by the analytical solutions. The largest difference between the analytical and numerical solutions appears to be the slower acceleration in the $z$ direction, which results in a smaller Doppler shift of the ions due to the directed expansion of the plasma.

It is possible that ion correlations are important to more quantitatively interpret this data. The decoupled isothermal assumption resulted in an anisotropic, Gaussian expansion. It may be possible that the particle correlations force the Gaussian profile as the system expands, and the effects of these correlations may result in an expansion whose details differ from the one offered in this model.

Making the plasmas more ideally two-dimensional should enable more careful tests of this model. It should further suppress effects due to expansion in the long dimension. Increasing the range of initial electron temperatures and plasma densities can probe interesting regions of phase space where differences between the data and the model are likely to be more pronounced. It is also possible to use the results of this isothermal model in more sophisticated treatments of the fluorescence signal, such as simulations numerically integrating the optical Bloch equations.

It should be possible to extend this model to include effects due to electron-ion recombination at early times. For strongly coupled neutral plasmas, the three-body recombination rate should be on the order of the plasma frequency. Using the high sensitivity and fast time response of fluorescence spectroscopy, it should be possible to directly measure spectroscopic recombination signatures in low-temperature, low-density plasmas, where the predicted three-body recombination rate is greater than the plasma frequency. For example, reducing the plasma density to $10^{6} \mathrm{~cm}^{-3}$ will make the recombination time $\sim 1 / \omega_{p}=20 \mathrm{~ns}$. Increasing the probelaser intensity to 100 times the saturation intensity will shorten the early rise time of the fluorescence signal to around $10 \mathrm{~ns}$. Optical pumping time will be comparable to the correlation-induced heating time, around $200 \mathrm{~ns}$.

\section{ACKNOWLEDGMENTS}

This research is supported in part by the Brigham Young University, the Research Corporation, and the National Science Foundation (Grant No. PHY-9985027). One of the authors (S.D.B.) also acknowledges the support of the Alexander von Humboldt Foundation.

${ }^{1}$ T. C. Killian, S. Kulin, S. D. Bergeson, L. A. Orozco, C. Orzel, and S. L. Rolston, Phys. Rev. Lett. 83, 4776 (1999).

${ }^{2}$ S. Kulin, T. C. Killian, S. D. Bergeson, and S. L. Rolston, Phys. Rev. Lett. 85, 318 (2000).

${ }^{3}$ T. C. Killian, M. J. Lim, S. Kulin, R. Dumke, S. D. Bergeson, and S. L. Rolston, Phys. Rev. Lett. 86, 3759 (2001).

${ }^{4}$ C. E. Simien, Y. C. Chen, P. Gupta, S. Laha, Y. N. Martinez, P. G. Mickelson, S. B. Nagel, and T. C. Killian, Phys. Rev. Lett. 92, 143001 (2004).

${ }^{5}$ Y. C. Chen, C. E. Simien, S. Laha, P. Gupta, Y. N. Martinez, P. G. Mickelson, S. B. Nagel, and T. C. Killian, Phys. Rev. Lett. 93, 265003 (2004).

${ }^{6}$ E. A. Cummings, J. E. Daily, D. S. Durfee, and S. D. Bergeson, Phys. Rev. Lett. 95, 235001 (2005).

${ }^{7}$ F. Robicheaux and James D. Hanson, Phys. Rev. Lett. 88, 055002 (2002).

${ }^{8}$ F. Robicheaux and James D. Hanson, Phys. Plasmas 10, 2217 (2003).

${ }^{9}$ S. G. Kuzmin and T. M. O'Neil, Phys. Rev. Lett. 88, 065003 (2002).

${ }^{10}$ S. G. Kuzmin and T. M. O'Neil, Phys. Plasmas 9, 3743 (2002).

${ }^{11}$ S. Mazevet, L. A. Collins, and J. D. Kress, Phys. Rev. Lett. 88, 055001 (2002).

${ }^{12}$ T. Pohl, T. Pattard, and J. M. Rost, Phys. Rev. A 70, 033416 (2004).

${ }^{13}$ T. Pohl, T. Pattard, and J. M. Rost, Phys. Rev. Lett. 94, 205003 (2005).

${ }^{14}$ S. D. Bergeson and R. L. Spencer, Phys. Rev. E 67, 026414 (2003).

${ }^{15}$ M. A. Furman, Am. J. Phys. 62, 1134 (1994).

${ }^{16}$ M. L. Citron, H. R. Gray, C. W. Gabel, and C. R. Stroud, Jr., Phys. Rev. A 16, 1507 (1977)

${ }^{17}$ Y. Liu, J. Lin, G. Huang, Y. Guo, and C. Duan, J. Opt. Soc. Am. B 18, 666 (2001).

${ }^{18}$ E. L. Raab, M. Prentiss, A. Cable, S. Chu, and D. E. Pritchard, Phys. Rev. Lett. 59, 2631 (1987).

${ }^{19}$ A. D. Ludlow, H. M. Nelson, and S. D. Bergeson, J. Opt. Soc. Am. B 18, 1813 (2001).

${ }^{20}$ R. W. P. Drever, J. L. Hall, F. V. Kowalski, J. Hough, G. M. Ford, A. J. Munley, and H. Ward, Appl. Phys. B 31, 97 (1983).

${ }^{21}$ F. Torabi-Goudarzi and E. Riis, Opt. Commun. 227, 389 (2003).

${ }^{22}$ R. Le Targat, J.-J. Zondy, and P. Lemonde, Opt. Commun. 247, 471 (2005).

${ }^{23}$ K. G. Libbrecht, R. A. Boyd, P. A. Willems, T. L. Gustavson, and D. K. Kim, Am. J. Phys. 63, 729 (1995).

${ }^{24}$ K. L. Corwin, Z.-T. Lu, C. F. Hand, R. J. Epstein, C. E. Wieman, Appl. Opt. 37, 3295 (1998). 